\title{
DISCRETE APPROXIMATION OF A TIME DELAY SYSTEM AND DELTA MODEL SPECTRUM
}

\author{
Tomáš Vyhlídal, Pavel Zítek
}

\author{
Centre for Applied Cybernetics, \\ Institute of Instrumentation and Control Engineering, \\ Czech Technical University in Prague, \\ Technická St. 4, 16607 - Praha 6, Czech Republic, \\ vyhlidal@fsid.cvut.cz, zitek@fsid.cvut.cz
}

\begin{abstract}
The properties of delta model approximations of time delay systems obtained by means of first and second order numerical methods are investigated. A particular attention is paid to the relationship between the spectra of continuous and corresponding delta model. Based on the convergence between the delta model and the Laplace transform of the continuous time model, the convergence of the trajectories of the delta model poles to the spectrum of continuous time model is illustrated in the application example. Copyright $\odot 2005$ IFAC
\end{abstract}

Keywords: time delay system, solution operator, delta model, system spectrum

\section{INTRODUCTION}

The delta transform has been developed as a united framework for describing both the linear continuous and the discrete dynamic systems (Middleton and Goodwin, 1990). The original aim of this transform was to build up a bridge between the traditional Laplace and $\mathrm{Z}$ transforms admitting the infinitesimal transition from a discrete to a continuous description. So far, a relatively weak attention has been paid to applying the delta transform to a sampled-data representation of time delay systems (TDS) where the discretization issues are more involved than in the usual linear delay-free models due to discrete representations of time delay segments. As the structure of TDS is concerned a significantly wider variability is to be expected and particularly the retarded and neutral systems are to be distinguished (Górecki, et al., 1989). In addition, one of the most specific properties of TDS is their infinite spectrum of eigenvalues. As the discrete approximation is applied to TDS the infinite spectrum is transformed to a finite spectrum the order of which depends on the sampling period and on the numerical method used for the discretization. As will be shown, for investigating the relationship between the spectrum of continuous system and its sampled-data approximation the delta transform is a proper tool.

Consider an autonomous TDS of the retarded type and of the following form

$$
\frac{d \mathbf{x}(t)}{d t}=\mathbf{A}_{0} \mathbf{x}(t)+\sum_{j=1}^{N} \mathbf{A}_{j} \mathbf{x}\left(t-\tau_{j}\right)
$$

where $\mathbf{x} \in \mathrm{R}^{n}$ is the vector of state variables, $\mathbf{A}_{j} \in \mathrm{R}^{n \times n}$, $j=0 . . N$, are the constant coefficient matrices and $\tau_{1}<\tau_{2}<\ldots<\tau_{N-1}<\tau_{N}$ are the time delays .

The state of system (1) is given by the vector of functional segments 


$$
\boldsymbol{x}_{t}(\vartheta)=\mathbf{x}(t+\vartheta)-T \leq \vartheta \leq 0
$$

in the Banach space $C=C\left([-T, 0], \mathrm{R}^{n}\right)$ provided with the supremum norm. The state $\boldsymbol{x}_{t}$ of system (1) at time $t$ is uniquely determined by the initial condition function $\varphi \in \mathcal{C}$ by the following formula

$$
\stackrel{T}{ }(t) \boldsymbol{\varphi}=\boldsymbol{x}_{t}
$$

where $T^{9}(t)$ is the solution operator of (1) (Hale and Verduyn Lunel, 1993).

\subsection{Features of TDS spectrum}

The spectrum of poles $s_{i}$ of system (1) is determined by the roots of the following characteristic equation

$$
M(s)=\operatorname{det}(s \mathbf{I}-\mathbf{A}(s))=0
$$

where

$$
\mathbf{A}(s)=\mathbf{A}_{0}+\sum_{j=1}^{N} \mathbf{A}_{j} \exp \left(-s \tau_{j}\right)
$$

Note that the exponential terms arise from Laplace transform of the time shifting of the state variables involved in (1). Also note that the spectrum of $T^{8}(t)$ is given by the exponential transform $\exp \left(s_{i} t\right)$, see (Hale and Verduyn Lunel, 1993). The spectrum of roots of quasipolynomial (3) is infinite with a specific character of the root distribution (Bellman and Cooke, 1963). An important feature of the spectrum of retarded system (1) is that the number of roots satisfying $\mathfrak{R}\left(s_{i}\right)>a, a \in \mathrm{R}$ is finite. The system spectrum tends to form a finite number of asymptotic chains with the following features: $\mathfrak{R}\left(s_{i}\right) \rightarrow-\infty, \quad\left|\mathfrak{I}\left(s_{i}\right) / \mathfrak{R}\left(s_{i}\right)\right| \rightarrow \infty \quad$ and $\left|\arg \left(s_{i}\right)\right| \rightarrow \pi / 2$ as $\left|s_{i}\right| \rightarrow \infty$. Thus, the stability and the system dynamics are determined by a finite number of rightmost poles.

\subsection{Numerical computing of the spectrum}

The rigthmost poles of (1) can be computed by the numerical methods based on discretization of the solution operator or on discretization of the infinitesimal generator of the semigroup, see (Engelborghs and Roose, 2002) (using Linear Multistep methods), (Breda, et al., 2004) (using RungeKutta methods). Note that in such methods, the quality of the approximation decays with increasing modules of the poles. An alternative to these approaches is to compute the roots of quasipolynomial $M(s)$ using a method developed for computing roots of analytic functions, see e.g. (Kravanja, 2002). A powerful tool for computing the roots of (3) is a quasipolynomial mapping based technique (Vyhlídal, 2003), (Vyhlídal and Zítek, 2003). Using this technique, it is possible to compute large number of roots within the same predefined accuracy. This allows us to investigate the whole character of the spectrum including the asymptotic features of the spectrum root chains (Zítek and Vyhlídal, 2004).

\section{DISCRETE MODELS OF TDS}

In order to obtain a plain form of the delta model, only first and second order numerical methods are used for the discretization of TDS. Discrete approximations of TDS using more advanced methods can be found in (Engelborghs and Roose, 2002) or (Breda, et al., 2004).

\subsection{Discrete approximation of TDS}

As the delay values cannot be expected as an integer multiples of the discretization step $\Delta t$, the delayed variables have to be expressed by applying an interpolation method. If the first order interpolation is considered, the following formula is used to interpolate the delay variables

$$
\begin{aligned}
\left.x_{i}\left(t-\tau_{j}\right)\right|_{t=k \Delta t} \cong\left(1-\mu_{j}\right) x_{i}\left(k-d_{j}\right)+ \\
\\
+\mu_{j} x_{i}\left(k-d_{j}+1\right), i=1,2, \ldots n
\end{aligned}
$$

where $d_{j}$ is an integer satisfying $d_{j} \Delta t \geq \tau_{j} \geq\left(d_{j}-1\right) \Delta t \quad$ and $\quad \mu_{j} \in[0,1]$ are the weighting coefficients. Thus, the right-hand side of (1) is to be substituted by

$$
\mathbf{A}_{0} \mathbf{x}(t)+\sum_{j=1}^{N} \mathbf{A}_{j} \mathbf{x}\left(t-\tau_{j}\right) \rightarrow f_{k}=\sum_{l=0}^{H} \tilde{\mathbf{A}}_{l} \mathbf{x}_{k-l}
$$

where $H=\max \left(d_{j}\right)$ and, according to (4), $\tilde{\mathbf{A}}_{l}$ are the linear combinations of particular matrices $\mathbf{A}_{j}$.

Applying the numerical method of the following specific form

$$
\mathbf{x}_{k+1}=\mathbf{x}_{k}+\Delta t\left(\beta_{-1} f_{k+1}+\beta_{0} f_{k}\right)
$$

where $\beta_{-1}+\beta_{0}=1$, the discrete approximation of (1) acquires the following generic form

$\mathbf{x}_{k+1}=\mathbf{x}_{k}+\Delta t\left(\beta_{-1} \sum_{l=0}^{H} \tilde{\mathbf{A}}_{l} \mathbf{x}_{k-l+1}+\beta_{0} \sum_{l=0}^{H} \tilde{\mathbf{A}}_{l} \mathbf{x}_{k-l}\right)$

For the Euler explicit method $\beta_{-1}=0, \beta_{0}=1$, Euler implicit method $\beta_{-1}=1, \beta_{0}=0$ and Trapezoidal method $\beta_{-1}=0.5, \beta_{0}=0.5$. 


\subsection{Discrete approximation of the solution operator}

Let the system state $\boldsymbol{x}_{t}$ be approximated by the discrete state $\boldsymbol{x}_{k}$ given by a sequence of the discrete state variables

$$
\boldsymbol{x}_{t} \rightarrow \boldsymbol{x}_{k}=\left[\begin{array}{lllll}
\mathbf{x}_{k,} & \mathbf{x}_{k-1}, & \ldots ., & \mathbf{x}_{k-H+1}, & \mathbf{x}_{k-H}
\end{array}\right]^{T}
$$

the discrete approximation of TDS (7) can be transformed into the form

$$
\boldsymbol{x}_{k+1}=\boldsymbol{\Phi} \boldsymbol{x}_{k}
$$

where

$$
\begin{gathered}
\boldsymbol{\Phi}=\left[\begin{array}{ccccc}
\mathbf{P Q}_{0} & \mathbf{P Q}_{1} & \ldots & \mathbf{P Q}_{H-1} & \mathbf{P Q}_{H} \\
\mathbf{I} & \mathbf{0} & \ldots & \mathbf{0} & \mathbf{0} \\
\mathbf{0} & \mathbf{I} & \ldots & \mathbf{0} & \mathbf{0} \\
\ldots & \ldots & \ldots & \ldots & \ldots \\
\mathbf{0} & \mathbf{0} & \ldots & \mathbf{I} & \mathbf{0}
\end{array}\right] \\
\mathbf{P}=\left(\mathbf{I}-\Delta t \beta_{-1} \tilde{\mathbf{A}}_{0}\right)^{-1}, \mathbf{Q}_{0}=\mathbf{I}+\Delta t\left(\beta_{-1} \tilde{\mathbf{A}}_{1}+\beta_{0} \tilde{\mathbf{A}}_{0}\right) \\
\mathbf{Q}_{m}=\Delta t\left(\beta_{-1} \tilde{\mathbf{A}}_{m+1}+\beta_{0} \tilde{\mathbf{A}}_{m}\right), m=1 . . H
\end{gathered}
$$

which is a discrete approximation of the equation

$$
\boldsymbol{x}_{t+\Delta t}=T^{9}(\Delta t) \boldsymbol{x}_{t}
$$

Similarly as in (2), the solution operator $T^{q}(\Delta t)$ maps $\boldsymbol{x}_{t}$ into $\boldsymbol{x}_{t+\Delta t}$. Thus, the matrix $\boldsymbol{\Phi}$ is a discrete approximation of the solution operator $T^{9}(\Delta t)$.

\section{DELTA MODEL AND ITS SPECTRUM}

The delta transform has been introduced by (Middleton and Goodwin, 1990) as an alternative to the Z-transform to analyze the dynamics of discrete time systems. The fundamental feature of the delta transform is that as $\Delta t \rightarrow 0$ the delta model of the system converges to its Laplace transform. The relation between the operators of the Z-transform $z$, the Laplace transform $s$ and the delta transform $\delta$ can be expressed by the following formula

$$
z=\exp (\Delta t s)=1+\Delta t \delta
$$

As $\Delta t \rightarrow 0$, according to the following limit

$$
\lim _{\Delta t \rightarrow 0} \delta=\lim _{\Delta t \rightarrow 0} \frac{\exp (s \Delta t)-1}{\Delta t}=s
$$

the operator $\delta$ converges to the operator $s$.

The model of system (1) can be considered in the following form

$$
\mathbf{X}(\delta)=I(\delta) \mathbf{F}(\delta)
$$

where $\mathbf{X}(\delta), \mathbf{F}(\delta)$ are the transforms of $\mathbf{x}(t)$ and of the right-hand side of the TDS approximated by (5), respectively, and $I(\delta)$ is a delta transfer function of the discrete time integrator, see (Comeau and Hori, 1997), (Zítek and Petrová, 2002). To obtain a delta model corresponding to (6), the delta transfer function is considered as follows

$$
I(\delta)=\frac{c_{0}+c_{1} \delta}{\delta}
$$

where $c_{0}$ and $c_{1}$ are continuous functions of $\Delta t$ $\left(c_{0}=\beta_{-1}+\beta_{0}\right.$ and $\left.c_{1}=\Delta t \beta_{-1}\right)$. The model (12) is a valid delta model of system (1) if

$$
\lim _{\Delta t \rightarrow 0} c_{0}=1 \text { and } \lim _{\Delta t \rightarrow 0} c_{1}=0
$$

see (Comeau and Hori, 1997). Taking into account that $(1+\Delta t \delta)$ represents a $\Delta t$ time shifting (Middleton and Goodwin, 1990), considering the zero initial conditions, the delta model of system (1) is given by

$$
\delta \mathbf{X}(\delta)=\left(c_{0}+c_{1} \delta\right)\left[\sum_{l=0}^{H} \tilde{\mathbf{A}}_{l}(1+\Delta t \delta)^{-l} \mathbf{X}(\delta)\right]
$$

which is also the delta transform of (7). Simultaneously, a delta transform of (8) and (9), respectively, can directly be expressed as

$$
\delta \boldsymbol{X}(\delta)=\frac{1}{\Delta t}(\boldsymbol{\Phi}-\mathbf{I}) \boldsymbol{X}(\delta)
$$

where $\boldsymbol{X}(\delta)$ is the delta transform of $\boldsymbol{x}_{k}$.

\subsection{Basic features of TDS delta model}

Order of TDS delta model. Unlike the case of a delay free linear system see (Comeau and Hori,1997), the order of the delta model of TDS given by (15) or (16) depends not only on $n$ (dimension of the state vector) and on the numerical method used, but also on the ration of the maximum system delay $\tau_{N}$ and $\Delta t$ as follows

$$
n_{\delta}=n\left(\operatorname{int}\left(\frac{\tau_{N}}{\Delta t}\right)+2\right)
$$

Convergence to Laplace transform model. As it is shown below, the convergence of the delta model to its Laplace transform as $\Delta t \rightarrow 0$ is preserved also for TDS. 
For the zero initial conditions, the Laplace transform of (1) is given by

$$
s \mathbf{X}(s)=\left[\mathbf{A}_{0}+\sum_{j=1}^{N} \mathbf{A}_{j} \exp \left(-s \tau_{j}\right)\right] \mathbf{X}(s)
$$

where $\mathbf{X}(s)$ is the Laplace transform of $\mathbf{x}(t)$. Taking into account (11) and (14) the delta transfer function (13) has the following limit

$$
\lim _{\Delta t \rightarrow 0} \frac{c_{0}+c_{1} \delta}{\delta}=\frac{1}{s}
$$

which is the Laplace transform of the continuous time integrator. The Laplace transform of a time shifting by $\tau_{j}$ results from its delta transform as the following limit

$$
\lim _{\Delta t \rightarrow 0}(1+\Delta t \delta)^{-\frac{\tau_{j}}{\Delta t}}=\exp \left(-s \tau_{j}\right)
$$

Decomposing the matrices $\tilde{\mathbf{A}}_{l}$ back into $\mathbf{A}_{j}$ according to (4) and (5) and taking into account (19) and (20), the delta model of TDS (15) converges to the Laplace transform (18) as $\Delta t \rightarrow 0$.

Stability region. The stability region in the $\delta$-plane is enclosed by a circle with a midpoint at $-\Delta t^{-1}$ and a diameter $2 \Delta t^{-1}$ (Middleton and Goodwin, 1990). Obviously, in the limit case, as $\Delta t \rightarrow 0$, in agreement with (11), the stability region covers the whole left half of the complex plane.

\subsection{Computing the delta model spectrum}

Spectrum as the polynomial roots. The first possibility to compute the spectrum of delta model of TDS given by (15) is to compute the roots of the delta model characteristic equation

$$
\begin{aligned}
Q(\delta)= & (1+\Delta t \delta)^{H} \operatorname{det}[\delta \mathbf{I}- \\
& \left.-\left(c_{0}+c_{1} \delta\right) \sum_{l=0}^{H} \tilde{\mathbf{A}}_{l}(1+\Delta t \delta)^{-l}\right]=0
\end{aligned}
$$

where $Q(\delta)$ is a polynomial. However, this approach is not safe because of the numerical reasons. It is due to the fact that the higher order polynomials, which are likely to be encountered in this case, are often illconditioned (Wilkinson, 1984).

Spectrum as the matrix eigenvalues. From the numerical point of view, it is much safer to compute the spectrum of the delta model as the eigenvalues of the matrix $\Delta t^{-1}(\boldsymbol{\Phi}-\mathbf{I})$ taken from (16). It allows us to handle the delta model even of a very high order using a relatively small $\Delta t$.

\subsection{Spectrum of the delta model}

As has been shown in (Comeau and Hori, 1998) the modes of the discretized model can be divided into two groups. The first group consists of modes with the counterparts in the dynamics of the continuous time model. The second group consists of modes introduced by the discretization process. Note that, as a rule, the modes in the second group are much faster than the modes in the first group.

In the $\delta$ - plane, provided that $\Delta t$ is chosen well according to the system dynamics, the poles introduced by the discretization are distributed close to the midpoint of the stability region while the poles with the counterparts in the continuous spectrum are distributed in a finite number of chains located rather close to the stability boundary circle. Transforming the poles of the delta model into the $s$-plane by the following formulas

$$
\begin{aligned}
& \operatorname{Re}\left(\hat{s}_{i}\right)=\frac{1}{\Delta t} \ln \left(\left|1+\Delta t \delta_{i}\right|\right), \\
& \operatorname{Im}\left(\hat{s}_{i}\right)=\arg \left(1+\Delta t \delta_{i}\right)=\frac{1}{\Delta t} \operatorname{atan}\left(\frac{\operatorname{Im}\left(1+\Delta t \delta_{i}\right)}{\operatorname{Re}\left(1+\Delta t \delta_{i}\right)}\right)
\end{aligned}
$$

which result from the mutual relation between the operators (10), those $\hat{s}_{j}$ which have the counterparts in the continuous time model are transformed by (22) into their vicinities. Note that the distances of the pairs $\hat{s}_{j}$ and $s_{j}$, which correspond each to other, are increasing with increasing modules of the poles. Thus, the dominant system modes are the best fitted ones. If the poles introduced by the discretization are transformed by (22) into the $s$ - plane, according to the fact that these poles are located close to the midpoint of the stability boundary of the $\delta$ - plane, it results from the first formula of (22) that these poles are transformed much farther to the left than the poles from the first group.

According to (17), as $\Delta t$ decreases from a certain starting value, the order of the delta model is gradually growing by factor $n$. Thus the number of poles in both groups of poles gradually increases. This feature will be illustrated in the following example. It will be shown that as the new poles emerge they become the poles of the second group. Simultaneously and also gradually, some of the poles leave the second group and along continuous trajectories become the poles with the counterparts in the continuous time model spectrum. It will also be illustrated that as $\Delta t$ continually decreases the poles of the delta model converge, along continuous trajectories, to the poles of continuous time model. 


\section{APPLICATION EXAMPLE}

A laboratory heating system, (Vyhlídal, 2003), is described by TDS model (1) with the following dynamics matrix

$$
\mathbf{A}(s)=\left[\begin{array}{cccc}
\frac{-\mathrm{e}^{-6.5 s}}{14} & \frac{0.24 \mathrm{e}^{-40 s}}{14} & 0 & 0 \\
\frac{0.5}{1.5} & \frac{-1}{1.5} & 0 & \frac{0.5 \mathrm{e}^{-13 s}}{1.5} \\
0 & \frac{0.94 \mathrm{e}^{-18 s}}{7} & \frac{-1}{7} & 0 \\
0 & 0 & \frac{0.81 \mathrm{e}^{-2.8 s}}{25} & \frac{-\mathrm{e}^{-9.2 s}}{25}
\end{array}\right]
$$

Using the trapezoidal rule for the discretization, a delta model of the form (16) is obtained. With respect to the shortest delay $\tau_{1}=2.8$ and the smallest time constant $T=1.5$, the sampling period is chosen $\Delta t=1.4$.

The spectrum of the continuous time TDS model with dynamics matrix (23) in the $s$-plane (black circles), the spectrum of the delta model in the $\delta$ plane (asterisks) and the delta model spectrum transformed to the s-plane by (22) (empty large circles) are seen in Fig. 1. Note that the poles of the continuous TDS are computed by mapping based

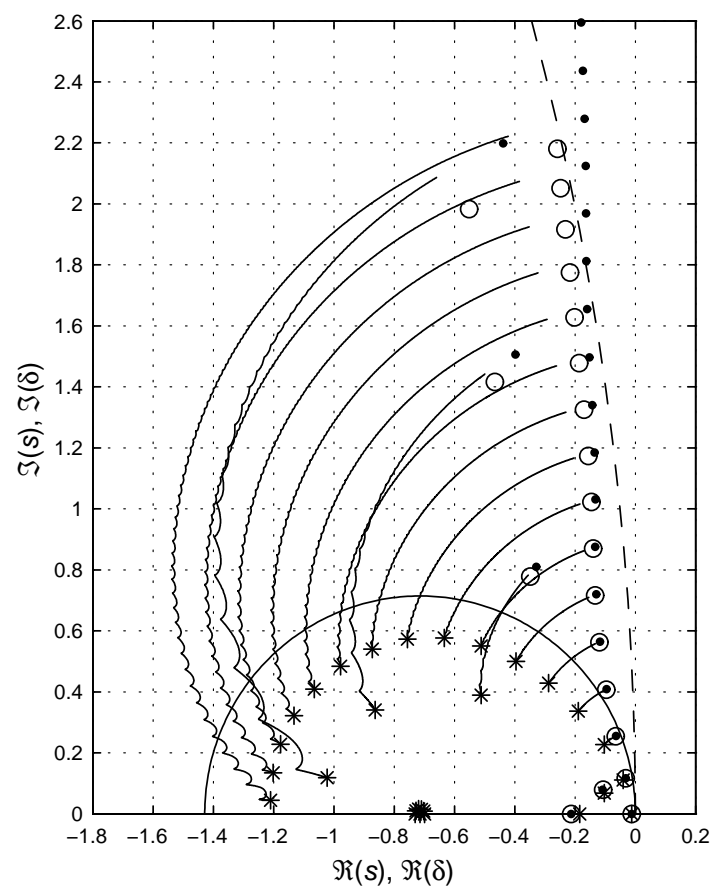

Fig. 1 Spectra of the continuous TDS (black circles) and its delta model (asterisks), (Trapezoidal method, $\Delta t=1.4$ ); empty circles - spectrum of the delta model transformed into the $s$-plane, trajectories of the poles of the delta model for $\Delta t \in[0.1,1.4]$, circle - stability boundary of $\delta$-plane for $\Delta t=1.4$, dashed circle - stability boundary of $\delta$-plane for $\Delta t=0.1$

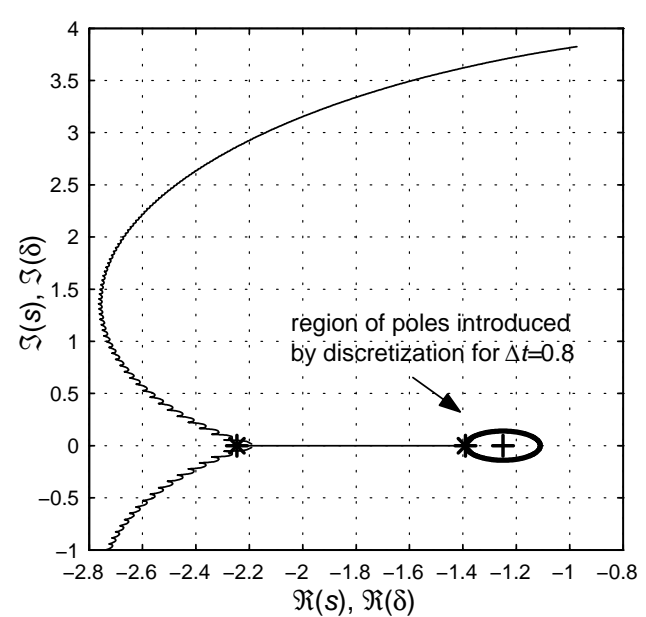

Fig. 2 The trajectories of a single couple of poles emerging from the region of poles introduced by the discretization for the discretization step decreasing from $\Delta t=0.8$ to $\Delta t=0.1$.

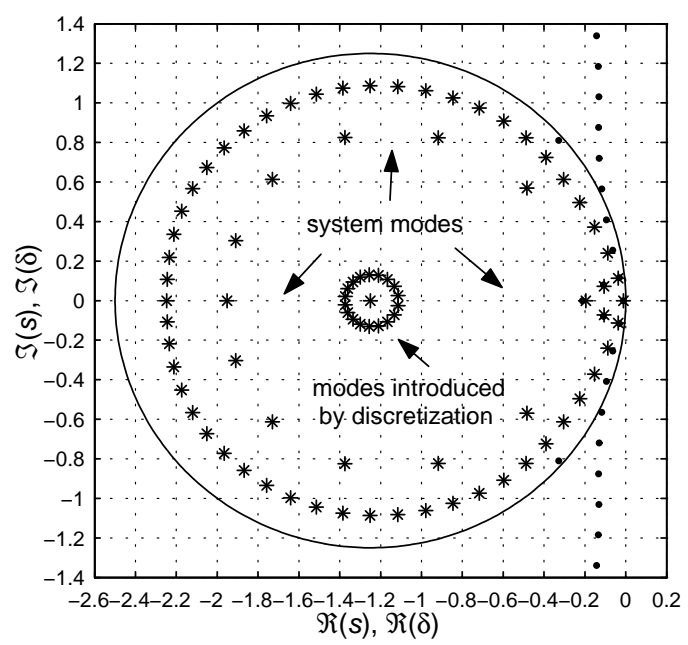

Fig. 3 Spectrum of delta model for $\Delta t=0.8$ (asterisks), spectrum of continuous TDS (black circles)

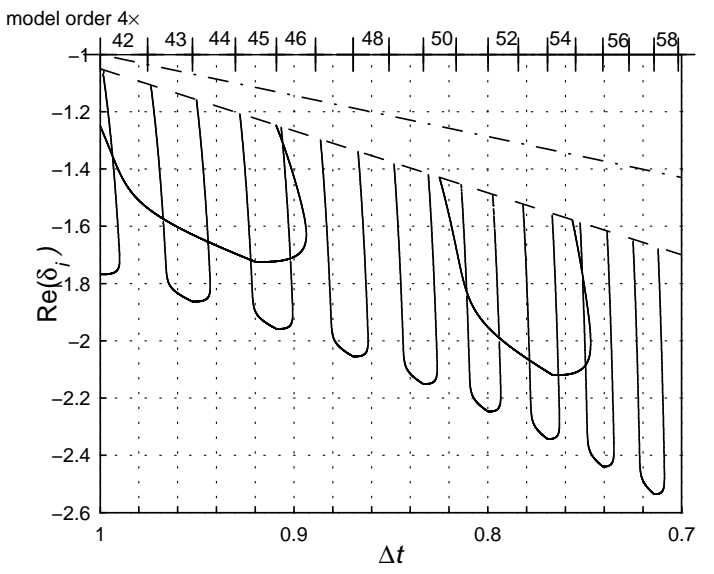

Fig. 4 The trajectories of the real poles of the delta model emerging from the region of poles introduced by the discretization, dahsed boundary of the region, dash-dotted - midpoint of the stability boundary circle. 
rootfinder (Vyhlídal and Zítek, 2003) while the eigenvalues of the large sparse matrix $\Delta t^{-1}(\boldsymbol{\Phi}-\mathbf{I}) \in \mathrm{R}^{120 \times 120}$ are computed using Lapac subroutines in Matlab. The curves also seen in Fig. 1 are the trajectories of the poles of the delta model with the discretization step continually decreasing from $\Delta t=1.4$ to $\Delta t=0.1$ (for which $n_{\delta}=1608$ ). As can clearly be seen from the course of the trajectories, as $\Delta t \rightarrow 0$, the trajectories will reach the positions of the continuous TDS poles.

Note that as $\Delta t$ decreases, gradually, from the region of poles introduced by the discretization process, additional poles emerge and along continuous trajectories become poles with the counterparts in the spectrum of the continuous time model. The trajectories of a single couple of poles are seen in Fig. 2 for $\Delta t$ decreasing from $\Delta t=0.8$ to $\Delta t=0.1$, see also the whole spectrum of the delta model for $\Delta t=0.8$ in Fig. 3. As can be seen, the poles leave the group of poles introduced by the discretization, one by one, as real poles. They gradually become the couples of poles and leave the real axis at the rightmost positions of the trajectories seen in Fig. 4.

\section{CONCLUSIONS}

The features of the delta model of TDS have been investigated. Even though only first and second order numerical methods were considered for the discretization, the results obtained reveal the fundamental properties of TDS delta models. It has been shown that as $\Delta t \rightarrow 0$, the delta model obtained converges to the Laplace transform of the system. The particular attention has been paid to the relationship between the spectrum of continuous TDS and the spectrum of its delta model. Next to the delta model poles which have the counterparts in the spectrum of TDS, the delta model spectrum involves a group of 'fast' poles, which are introduced by the discretization process. The movement of the poles subject to decreasing discretization step has been demonstrated in the application example. It has been shown that as the delta model order increases, the poles are introduced into the group of fast poles and then, gradually, on the continuous trajectories, they become the poles which have the counterparts in the continuous TDS spectrum. It has also been shown that the rightmost poles of TDS are covered with the highest accuracy by the corresponding poles in the discretized model spectrum. To sum up, it has been shown that the delta transform can also be utilized in the analysis of TDS dynamics, e.g. in the process of selecting suitable $\Delta t$ for which the dominant continuous TDS modes are sufficiently covered by the discretized model.

\section{ACKNOWLEDGEMENT}

The presented research has been supported by the Ministry of Education of the Czech Republic under the Project 1M6840770004.

\section{REFERENCES}

Bellman, R., and Cooke, K.L. (1963), Differentialdifference equation, Academic Press, New York.

Breda, D., Maset, S. and Vermiglio, R. (2004). Computating the characteristic roots for delay differential equations, IMA Journal of Numerical Analysis, vol. 24, pp. 1-19.

Comeau, A. R. and Hori, N., (1998), State-space forms for higher-order discrete-time models, Systems \& Control Letters, vol. 34, pp. 23-31.

Hale, J. K., and Verduyn Lunel, S. M., (1993), Introduction to functional differential equations, Vol. 99 of Applied Mathematical Sciences, Springer Verlag New York Inc

Engelborghs, K., Roose, D. (2002), On stability of LMS-methods and characteristic roots of delay differential equations, SIAM Journal of Numerical Analysis, Vol. 40, No. 2, pp. 629-650.

Górecki, H., S. Fuksa, P. Grabowski and A. Korytowski (1989), Analysis and synthesis of time delay systems, Polish Scient. Publ. Warszawa.

Kravanja, P. and Barel, M.V. (2000). Computing the zeros of analytic functions. Lecture Notes in Mathematics, Vol. 1727, Springer.

Middleton and Goodwin, (1990), Digital control and estimation, Prentice-Hall.

Vyhlídal, T. (2003), Analysis and synthesis of time delay system spectrum, Ph.D. Thesis, Faculty of Mechanical Engineering, Czech Technical University in Prague.

Vyhlídal, T. and Zítek, P., (2003), Quasipolynomial mapping based rootfinder for analysis of Time delay systems, In Proc IFAC Workshop on TimeDelay Systems, TDS'03, Rocquencourt

Wilkinson, J. H. (1984), The perfidious polynomial, Studies in Numerical Analysis, M.A.A. Studies in Mathematics, 24, Gene H. Golub, ed., pp. 128, 1984.

Zítek, P. and Petrová, R. (2002), Discrete approximation of anisochronic systems using delta transform, In Proc. of Process Control - Rip 2002. Pardubice, University of Pardubice.

Zítek, P. and Vyhlídal, T., (2004), A Residue Based Evaluation of Pole Significance in Time Delay Systems, In Proc IFAC Workshop on TimeDelay Systems, TDS'04, Leuven. 